\title{
THE GRADED CARTAN MATRIX AND GLOBAL DIMENSION OF 0-RELATIONS ALGEBRAS
}

\author{
by W. D. BURGESS*
}

(Received 4th September 1985)

\section{Introduction}

The Cartan matrix $C$ of a left artinian ring $A$, with indecomposable projectives $P_{1}, \ldots, P_{n}$ and corresponding simples $S_{i}=P_{i} / J P_{i}$, is an $n \times n$ integral matrix with entries $c_{i j}$, the number of copies of the simple $s_{j}$ which appear as composition factors of $P_{i}$. A relationship between the invertibility of this matrix (as an integral matrix) and the finiteness of the global dimension has long been known: $\operatorname{gl} \operatorname{dim} A<\infty \Rightarrow \operatorname{det} C= \pm 1$ (Eilenberg [3]). More recently Zacharia [9] has shown that $\operatorname{gl} \operatorname{dim} A \leqq 2 \Rightarrow \operatorname{det} C=1$, and in fact no rings of finite global dimension are known with $\operatorname{det} C=-1$. The converse, $\operatorname{det} C=1 \Rightarrow \mathrm{gl} \operatorname{dim} A<\infty$, is false, as easy examples show ([1) or [3]). However if $A$ is left serial, gl $\operatorname{dim} A<\infty$ iff $\operatorname{det} C=1$ [1]. If $A=\bigoplus_{n \geqq 0} A_{n}$ is $\mathbb{Z}$-graded and the radical $J=\bigoplus_{n \geqq 0} A_{n}$, Wilson [8] calls such rings positively graded. Here there is a graded Cartan matrix $\tilde{C}$ with entries from $\mathbb{Z}[X]$ and $\operatorname{gl} \operatorname{dim} A<\infty \Rightarrow \operatorname{det} \tilde{C}=1$ and, hence, $\operatorname{det} C=1$ [8, Prop. 2.2].

The purpose of this note is to investigate the converse of Wilson's result for 0 relations algebras. Green, Happel and Zacharia [7] have devised a method for calculating the global dimension of 0 -relations algebras by looking at how the relations overlap along possibly infinite paths in the quiver. If the quiver of a 0 -relations algebra $A$ has $S$ arrows then it is shown below that $A$ has a natural positive $\mathscr{G}$-grading, where $\mathscr{G}$ is the free group on $s$ generators. This also gives a $G$-grading where $G$ is the free abelian group on $s$ generators. The corresponding Cartan matrices, $\hat{C}$ and $H$, have entries in $\mathbb{Z}\left\langle x_{1}, \ldots, x_{s}\right\rangle$ and $\mathbb{Z}\left[x_{1}, \ldots, x_{s}\right]$, respectively. It is shown that $\operatorname{gl} \operatorname{dim} A<\infty$ iff $\hat{C}$ is invertible, and that $\mathrm{gl} \operatorname{dim} A<\infty \Rightarrow \operatorname{det} H=1$. Unfortunately, while $\hat{C}$ is easy to find, its invertibility is hard to test. It is conjectured that the converse of the latter result is true and the remainder of the paper examines special cases. If the relations ideal giving $A$ can be generated by paths of length 2 and the Loewy length of $A$ is $\leqq 3$, then $\operatorname{gl} \operatorname{dim} A<\infty$ iff $\operatorname{det} H=1$. This is shown to give all the 0-relations algebras of Loewy length $\leqq 3$ when the quiver has $\leqq 3$ vertices, but not for more vertices.

The author would like to thank K. R. Fuller and B. Zimmermann-Huisgen for showing him some unpublished work on Cartan matrices and $\mathbb{Z}$-graded rings, and Fuller for some valuable discussions.

\section{1.}

We begin by recalling the outlines of the calculus in [7]. Given a quiver (directed graph) $Q$ and a field $K$, a path algebra $K Q$ can be constructed (e.g. [5]). A 0-relations

*The author gratefully acknowledges the support of NSERC Grant A7539. 
algebra $A$ is a finite dimensional quotient $A=K Q / I$, where $I$ is generated by a collection $\rho$ of paths, called relations, of length $\geqq 2$. A generating set will always be taken to be minimal, i.e., no proper subset of $\rho$ will generate $I$.

A path in $Q$ will always be oriented and repetitions of vertices or arrows are allowed. Given a path $P$ (possibly infinite)

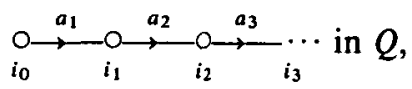

the associated sequence of relations, $R$, is defined as follows [7, p. 184]: if no relation of $\rho \cap P$ has initial point $i_{0}$, then $R=\phi$, if $r_{1} \in \rho \cap P$ and its initial point $s\left(r_{1}\right)=i_{0}$ then $r_{1} \in R$; if some $r \in \rho$ has $s(r)<e\left(r_{1}\right)$, end point of $r_{1}$ (the symbol "<" is read as "before") then let $r_{2}$ be one such with least initial point and then $r_{2} \in R$, if there is none such, $R=\left\{r_{1}\right\}$; if $r_{1}, \ldots, r_{m} \in R$ and some $r \in \rho \cap P$ has $e\left(r_{m-1}\right) \leqq s(r)<e\left(r_{m}\right)$ then put $r_{m+1}$ to be the one with least initial point and $r_{m+1} \in R$, if there is none such, $R=\left\{r_{1}, \ldots, r_{m}\right\}$.

Given a vertex $i_{0}$, the projective dimension of the corresponding simple $S_{i_{0}}$ is $\sup _{P_{E} \mathscr{P}}\{($ cardinality of the associated sequence of $P)+1\}$ where $\mathscr{P}$ is the collection of paths beginning at $i_{0}[7,1.2$ and 2.3$]$, if $\mathscr{P} \neq \varnothing$, otherwise $S_{i_{0}}$ is projective.

Proposition 1.1 Let $A=K Q / I$ be a 0-relations algebra. Then if $Q$ has $s$ arrows $A$ has a $\mathscr{G}$-grading where $\mathscr{G}$ is the free group on $s$ generators (in fact only non-negative exponents are used so it could be considered a free monoid grading). This grading induces a Ggrading where $G$ is the free abelian group on senerators.

Proof. $A$ is spanned as a vector space by the non-zero paths of $Q$. A non-zero path $a_{i_{1}}, \ldots, a_{i_{m}}$ is decreed to be of degree $x_{i_{1}} \ldots x_{i_{m}}$. That this is a grading is obvious.

If all the $x_{i}$ are sent to some new variable $y$, the above grading induces a $\mathbb{Z}$-grading on $A$ which makes $A$ positively graded in the sense of Wilson.

(A special case of the gradings investigated by Green [6] shows that every 0 -relations algebra is graded by a free group which is the fundamental group of the quiver viewed as an unoriented graph. In these gradings some radical elements may be of degree 1 , the neutral element of the group, and, hence, they serve less well for our calculations than the gradings defined above.)

To each of these gradings of $A$ there corresponds a Cartan matrix. For the $\mathscr{G}$-grading, the entries of the Cartan matrix $\hat{C}$ are from the free ring $\mathbb{Z}\left\langle x_{1}, \ldots, x_{s}\right\rangle$, while the $G$ grading yields a Cartan matrix, $H$, with entries from $\mathbb{Z}\left[x_{1}, \ldots, x_{s}\right]$. If the vertices of $Q$ are labeled $1,2, \ldots, n$ then $\hat{C}$ is an $n \times n$ matrix with $i j$ entry a sum of monomials, a monomial $x_{i_{1}} \ldots x_{i_{m}}$ appearing for every copy of the simple $S_{j}$ found in a graded composition series for $R e_{i}$ in degree $x_{i_{1}} \ldots x_{i_{m}} ; H$ is defined analogously.

This is best illustrated by a simple example.

Example 1.2.

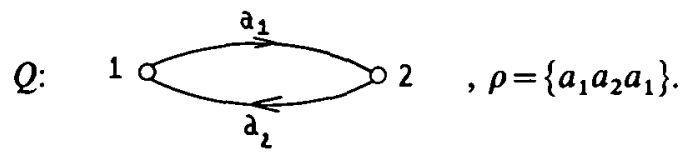


$A$ is spanned by $\left\{e_{1}, e_{2}, a_{1}, a_{2}, a_{1} a_{2}, a_{2} a_{1}, a_{2} a_{1} a_{2}\right\}$. The $\mathscr{G}$-grading is: $A_{1}=\left\{K e_{1}+K e_{2}\right\}$, $A_{x_{1}}=\left\{K a_{1}\right\}, A_{x_{2}}=\left\{K a_{2}\right\}, A_{x_{1} x_{2}}=\left\{K a_{1} a_{2}\right\}, A_{x_{2} x_{1}}=\left\{K a_{2} a_{1}\right\}, A_{x_{2} x_{1} x_{2}}=\left\{K a_{2} a_{1} a_{2}\right\}$. (The $G$-grading would put $A_{x_{1} x_{2}}$ and $A_{x_{2} x_{1}}$ together.)

The Cartan matrices are:

$$
\hat{C}=\left[\begin{array}{cc}
1+x_{1} x_{2} & x_{2}+x_{2} x_{1} x_{2} \\
x_{1} & 1+x_{2} x_{1}
\end{array}\right], \quad H=\left[\begin{array}{cc}
1+x_{1} x_{2} & x_{2}+x_{1} x_{2}^{2} \\
x_{1} & 1+x_{1} x_{2}
\end{array}\right]
$$

It can be seen that $\hat{C}$ completely determines $A$.

An argument first used by Eilenberg [3] for ungraded algebras and adapted by Wilson [8] to the positively graded case works equally well here. It is clear that the simple modules, the indecomposable projectives and the radical of $\boldsymbol{A}$ are all gradable modules, i.e., can be given the structure of $\mathscr{G}$ - or $G$-graded modules.

Proposition 1.3. Let $A=K Q / I$ be a 0-relations algebra graded by the free group $\mathscr{G}$ (free abelian group $G$ ). If $\mathrm{gl} \operatorname{dim} A<\infty$ then the $\mathscr{G}$-graded Cartan matrix is invertible over $\mathbb{Z}\left\langle x_{1}, \ldots, x_{s}\right\rangle$ (the G-graded Cartan matrix has determinant 1 ).

The essence of the argument is given below in proving a converse in the $\mathscr{G}$-graded case. The result [7, Theorem 2.3] can be translated into a statement about $\hat{C}$. The $\mathscr{G}$ graded projective resolutions of the simples (in degree 1) will always yield a left inverse of $\hat{C}$, but with entries in the ring of formal power series, $\left.\mathbb{Z} \ll x_{1}, \ldots, x_{s}\right\rangle$. This occurs as follows. Consider the following ungraded projective resolution of $S_{i}$ :

$$
(R) \cdots \rightarrow Q_{i r} \rightarrow \cdots \rightarrow Q_{i 1} \rightarrow Q_{i 0} \rightarrow S_{i} \rightarrow 0
$$

where the $Q_{i j}$ are projectives. Since all the modules here are gradable, by taking the indecomposable components of $Q_{i j}$ in appropriate degrees, $(R)$ can be made into a graded resolution. The formal left inverse of $\hat{C}$ is formed along the lines of Wilson's computation. The resolution gives an alternating sum for each $k=1, \ldots, n$; here $\mathscr{M}$ is the set of elements of $\mathscr{G}$ formed from the $x_{i}$ (corresponding to the arrows $a_{i}$ ) using only nonnegative exponents:

$$
\sum_{r \in N} \sum_{j=1}^{n} \sum_{m \in \mathscr{N}}(-1)^{r} u(i, j, r, m) m \hat{c}_{j k}=\delta_{i k}
$$

where $u(i, j, r, m)$ is the number of occurrences in $Q_{i r}$ of the indecomposable projective $P_{j}$ in degree $m$, and $\hat{c}_{j k}$ is the $j k$ entry of $\hat{C}$. The $i j$ entry of the left inverse of $\hat{C}$ is thus

$$
\sum_{r \in N} \sum_{m \in \mathscr{M}}(-1)^{r} u(i, j, r, m) m=\hat{d}_{i j}
$$

Now $u(i, r, j, m) \neq 0$ iff $P_{j}$ occurs in degree $m$ in $Q_{i r}$ iff (by [7, Proposition 1.1 and Theorem 1.2]) there is a path from $i$ to $j$ in $Q$ using the arrows $a_{i}, \ldots, a_{i_{p}}$ (in order), where $m=x_{i_{1}} \ldots x_{i_{p}}$, whose associated sequence has $r$ steps and ends in $j$. 
Now two distinct paths correspond to distinct monomials so there can be no cancellation in the terms making up $\hat{d}_{i j}$. It follows that the left inverse matrix has entries which are in $\mathbb{Z}\left\langle x_{1}, \ldots, x_{s}\right\rangle$ iff $g l \operatorname{dim} A<\infty$. This completes the translation.

Proposition 1.4. Let $A=K Q / I$ be a 0 -relations algebra graded by the free group $\mathscr{G}$, then $\operatorname{gl} \operatorname{dim} A<\infty$ iff the graded Cartan matrix $\hat{C}$ has a inverse with coefficients in $\mathbb{Z}\left\langle x_{1}, \ldots, x_{s}\right\rangle$.

The difficulty here is that although it is easy to form $\hat{C}$, it is not easy to test if it has an inverse, although there are procedures for this. In the discussion above only a left inverse for $\hat{C}$ is constructed, but since $\mathbb{Z}\left\langle x_{1}, \ldots, x_{s}\right\rangle$ can be embedded in a division ring ([2], Corollary p. 80 and p. 283]), left-invertibility and invertibility coincide. Moreover the last step of the above argument does not apply to the $G$-grading; two distinct paths may have the same $G$-degree. All the examples tested to date suggest that indeed 1.4 would remain true if $\mathscr{G}$ were replaced by $G, \hat{C}$ by $H$ and the criterion by "Det $H=1$ ". The remainder of the paper shows this to be true for a special class of 0 -relations algebras.

This section will close with a remark about artinian rings in general. It will be used in the next section (2.8). The purpose is to show that for questions about the finiteness of the global dimension, there is no loss in generality in supposing that the quiver is strongly connected.

Definition 1.5. Let $Q$ be a graph. (i) $Q$ is said to be strongly connected if for any pair of vertices $i$ and $j$ there is a path from $i$ to $j$. (ii) There is an equivalence relation on the set of vertices given by $i \sim j$ if there are paths from $i$ to $j$ and from $j$ to $i$; the equivalence classes are called the strongly connected components. (iii) A set of vertices $S$ of $Q$ is called a sink if there is no arrow from a vertex in $S$ to a vertex not in $S$.

Proposition 1.6. Let $A$ be a basic left artinian ring and $Q$ its left quiver. If $S$ is a set of vertices which is a sink, denote by $e_{1}, \ldots, e_{m}$ the primitive idempotents corresponding to the vertices in $S, e=e_{1}+\cdots+e_{m}$. Then $\operatorname{gl} \operatorname{dim} A<\infty$ iff $\mathrm{gl} \operatorname{dim} e A e$ and $\operatorname{gl} \operatorname{dim}(1-e(A(1-e)<\infty$.

Proof. The result follows immediately from [4, Corollary 3.6] once one observes that $e A(1-e)=0$, and this is because $A(1-e)$ contains none of the simples associated with $e_{1}, \ldots, e_{m}$ as composition factors.

Any graph $Q$ must have a strongly connected component which is a sink, which allows a reduction to the strongly connected case.

\section{2.}

In this section we consider the graded Cartan matrix $H$. The aim is to show that for a special class of 0 -relations algebras, det $H=1$ implies finite global dimension. To begin there are some general remarks.

Lemma 2.1. Let $A$ be any 0-relations algebras. For any subset $S=\left\{a_{r_{1}}, \ldots, a_{r_{k}}\right\}$ of the 
set of arrows, form the 0-relations algebra just using the arrows of $S$ and the relations from $\rho$ involving only these arrows; call it $A^{\prime}$. Then the Cartan matrix $H^{\prime}$ of $A^{\prime}$ is obtained from $H$ by setting to zero all the variables other than $x_{r_{1}}, \ldots, x_{r_{k}}$. Further, if $\operatorname{det} H=1$ then $\operatorname{det} H^{\prime}=1$.

Proof. The simple $S_{j}$ appears in $A e_{i}$ in degree $x_{1}^{p_{1}} \ldots x_{m}^{p_{m}}$ exactly when there is a nonzero path from $i$ to $j$ using the arrow $a_{r}$ exactly $p_{r}$ times, $r=1, \ldots, m$. The same is true for $A^{\prime}$ with the arrows restricted to $S$. In other words, $H^{\prime}$ is obtained from $H$ by setting to zero all variables other than those corresponding to arrows in $S$. The last statement is now obvious.

Lemma 2.2. Suppose $A=K Q / I$ is a 0 -relations algebras for which $\operatorname{det} H=1$. Then $Q$ has no loops.

Proof. Suppose there is a loop $a$ corresponding to a generator $x$ of $G$. If $A^{\prime}$ is the algebra, as in (2.1), given by $a$ alone, then $H^{\prime}=\left[1+x+\cdots+x^{k}\right]$ for some $k \geqq 1$. Clearly $\operatorname{det} H^{\prime} \neq 1$, a contradiction by (2.1).

We now restrict our attention to a special class of algebras where $\rho$ consists of paths of length 2 . In this case the associated sequence of a path in $Q$ has relations starting on successive vertices, until it stops. That is, if we have a path

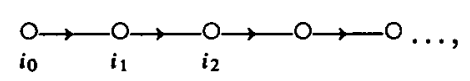

and the associated sequence has $N$ steps, the relations in it have initial points $i_{0}, i_{1}, \ldots, i_{N-1}$ and end points $i_{2}, i_{3}, \ldots, i_{N+1}$. This facilitates computations.

Lemma 2.3. Let $A=K Q / I$ where $I$ is generated by $\rho, \rho$ a set of paths of length 2 . Then $\mathrm{gl} \operatorname{dim} A=\infty$ iff there is a path

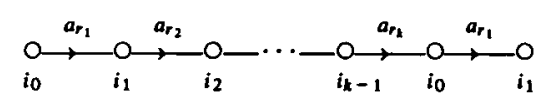

with $a_{r_{p}} a_{r_{p+1}}=0, p=1, \ldots, k-1, a_{r_{k}} a_{r_{1}}=0$.

Definition 2.4. Suppose $Q$ is a quiver and $\rho$ a set of paths of length 2. A path

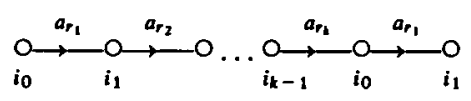

such that $a_{r_{p}} a_{r_{p+1}}=0, p=1, \ldots, k-1$ and $a_{r_{k}} a_{r_{1}}=0$, is called a shortest null cycle if there are none such with fewer arrows.

The aim is to show that if $\rho$ consists of paths of length 2 and the Loewy length is $\leqq 3$ then a shortest null cycle always gives a Cartan matrix of determinant $\neq 1$. 
The first observation about a shortest null cycle is that it can have no repeated arrows. Hence the following notation can be used:

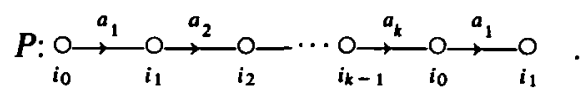

In future references to $P$ the indices of the arrows and corresponding generators of $G$ are always taken modulo $k$, with representatives $1,2, \ldots, k$.

Lemma 2.5. Let $A=k Q / I$ be of Loewy length $\leqq 3$ and such that $\rho$ may be chosen to consist of paths of length 2. Assume that det $H=1$ and that there is a shortest null cycle $P$. Then along $P$

(i) if $a_{r} a_{s}$ makes sense and $s \neq r+1$ or $r=k$ and $s \neq 1$, then $a_{r} a_{s} \neq 0$.

(ii) if a vertex $j$ appears more than once among $i_{0}, i_{1}, \ldots, i_{k-1}$ ( $j$ is then called a multiple vertex for $P$ ), then the vertices $\ell$ and $m$ in

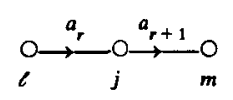

are distinct and neither appears again in $P$.

Proof. (i) If $a_{r} a_{s}=0$ with $s \neq r+1$ or $r=k$ and $s \neq 1$, then $P$ could be shortened by removing the arrows between $a_{r}$ and $a_{s}$.

(ii) Since there are no loops in $Q(2.2), \ell \neq j$ and $m \neq j$.

Suppose $\ell=m$. Then we have

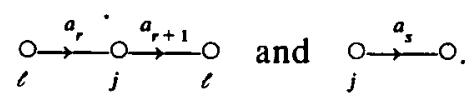

Since the Loewy length is $\leqq 3, a_{r+1} a_{r} a_{s}=0$ which implies that $a_{r+1} a_{r}=0$ or $a_{r} a_{s}=0$ (by the hypothesis on $\rho$ ). Both possibilities are excluded by (i).

Next suppose $\ell$ appears again. We have

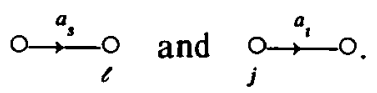

Then $a_{s} a_{r} a_{t}=0$, which is impossible unless $s=r-1$. A possible repetition of $m$ is dealt with similarly.

Theorem 2.6. Let $A=k Q / I$ be a 0 -relations algebra of Loewy length $\leqq 3$ such that $I$ is generated by a set $\rho$ consisting of paths of length 2 . Then if $H$ is the G-graded Cartan matrix, $\operatorname{det} H=1$ iff $\mathrm{gl} \operatorname{dim} A<\infty$. Further, if $s$ is the number of arrows and $N$ the cardinality of $\rho, \operatorname{gl} \operatorname{dim} A<\infty$ implies $\operatorname{gl} \operatorname{dim} A \leqq \min \{s, N+1\}$. 
Proof. One direction is already available. The proof of the converse is by contradiction. Suppose that $\operatorname{gl} \operatorname{dim} A=\infty$ and $\operatorname{det} H=1$. There is a shortest null cycle $P$ (by 2.3). We shall examine the matrix $H^{\prime}$ given by $P$ (as in 2.1 ); $\operatorname{det} H^{\prime}=1$.

The vertices of $P$ correspond to the rows and columns of $H^{\prime}$. The entries come from non-zero paths, including "paths" of length zero which give the constant 1 in each diagonal entry. There are two possibilities for a con-constant term in the $m \ell$ entry, a degree one term $x_{r}$ if there is an arrow

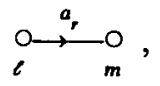

and a degree two term $x_{s} x_{t}$ if there is a non-zero path

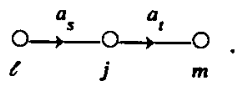

In the latter case, $j$ is necessarily a multiple vertex (as in 2.5(ii)). According to (2.5(ii)), the two cases cannot occur in the same entry and no entry can have more than one non-constant term. However a diagonal entry of the form $1+x_{u+1} x_{u}$ is possible coming from a configuration

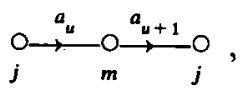

it is in the $m m$ entry.

Let us examine a row, say the $m$ th, which has a degree 2 entry arising from

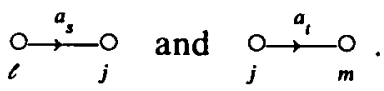

Note that $a_{t}$ is the only arrow ending in $m$. Further $j$ is a multiple vertex so that there is another arrow ending in $j$; there is

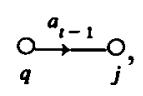

and there may be others, let

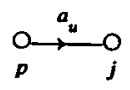

represent a typical one. Consider also the $j$ th row. Since vertices of $P$ adjacent to $j$ are 
not multiple, there are no degree 2 entries in the $j$ th row. In the illustration below any entry not listed is zero.

$$
\begin{aligned}
& \begin{array}{llllll}
\text { columns: } & p & \ell & q & m & j
\end{array} \\
& \begin{array}{llllll}
x_{u} x_{t} & x_{s} x_{t} & 0 & 1 & x_{t} & n \text {th row }
\end{array} \\
& \begin{array}{llllll}
x_{u} & x_{s} & x_{t-1} & 0 & 1 & j \text { th row }
\end{array}
\end{aligned}
$$

Now subtract $x_{t}$ times the $j$ th row from the $m$ th. We obtain

$$
\begin{array}{cccccc}
p & \ell & q & m & j & \\
0 & 0 & -x_{t-1} x_{t} & 1 & 0 & m \text { th }
\end{array}
$$

(A degree 2 diagonal entry $1+x_{u} x_{u+1}$ would be converted to 1 by this process.) If this is done for each row with degree 2 entries we get a new matrix, $K$. Of course $\operatorname{det} K=\operatorname{det} H^{\prime}$. A row of $K$ has at most one degree 2 entry, and it would be of the form $-x_{t-1} x_{t}$.

Each arrow of $P$ either appears in a configuration

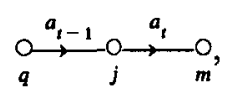

where $j$ is a multiple vertex, or as

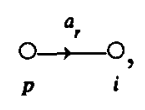

where $p$ and $i$ are simple vertices. This shows there is a term of $\operatorname{det} K$ of the form $\pm x_{1} x_{2} \ldots x_{k}$. It is obtained by using all the degree 2 entries from $K$ and the degree 1 entries corresponding to arrows whose end vertices are simple. Any unused columns and rows correspond to multiple vertices. From these the entry 1 is taken from the diagonal.

Next, no other term of det $K$ has the form $\pm x_{1} x_{2} \ldots x_{k}$. To see this note that if $a_{t}$ has initial vertex which is a multiple vertex, $x_{t}$ only appears in the degree 2 entry $-x_{t-1} x_{t}$. Similarly if the end vertex of $a_{u}$ is a multiple vertex, $x_{u}$ only appears in the degree 2 entry $-x_{u} x_{u+1}$. If both vertices of $a_{r}$ are simple, $x_{r}$ only appears in degree 1 . Hence there is only one way of obtaining $\pm x_{1} x_{2} \ldots x_{k}$.

The argument works vacuously when $P$ has no multiple vertices, and, hence, $H^{\prime}=K$ has no degree 2 entries.

Hence det $H^{\prime} \neq 1$ and we have a contradiction. The last statement of the theorem follows immediately from $[7,1.2]$.

The remainder of the article is to look at two special cases, where $Q$ has 2 or 3 vertices. The first lemma does not require any assumptions on $\rho$ or the Loewy length.

Lemma 2.7. Let $A=K Q / I$ be any 0 -relations algebra. If $Q$ contains a cycle of length 2 ,

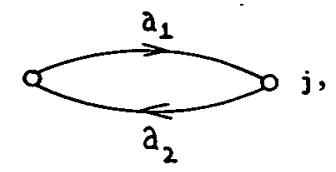
and $\operatorname{det} H=1$, then exactly one of $a_{1} a_{2}$ or $a_{2} a_{1}$ is 0 . 
Proof. The two arrows give a term $-x_{1} x_{2}$ in det $H$. It must be cancelled by $x_{1} x_{2}$ which can only come from $a_{1} a_{2} \neq 0$ and $a_{2} a_{1}=0$ (giving $1+x_{1} x_{2}$ in (ii) or $a_{1} a_{2}=0$ and $a_{2} a_{1} \neq 0$.

Proposition 2.8. Let $A=K Q / I$ be a 0 -relations algebra where $Q$ has 2 vertices. Then gl $\operatorname{dim} A<\infty$ iff $\operatorname{det} H=1$. If $\mathrm{gl} \operatorname{dim} A<\infty$ then $\mathrm{gl} \operatorname{dim} A \leqq 2$.

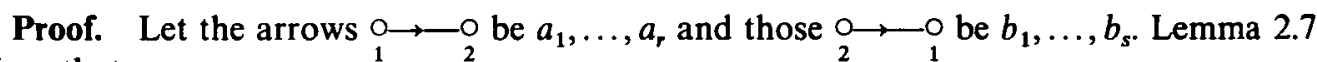
gives that

$$
H=\left[\begin{array}{cc}
1+\sum_{a_{i} b_{j} \neq 0} x_{i} y_{j} & \sum y_{j} \\
\sum x_{i} & 1+\sum_{a_{i} b_{j}=0} x_{i} y_{j}
\end{array}\right] .
$$

If $\operatorname{det} H=1$ then either all $a_{i} b_{j} \neq 0$ or all $a_{i} b_{j}=0$. In either case $A$ is clearly of global dimension $\leqq 2$, since in the former case, for example, the indecomposable projective $P_{1}$ has radical which is a direct sum of $s$ copies of $P_{2}$.

Notice that in the above setting, $\operatorname{det} H=1$ implies that $\rho$ may be taken to consist of paths of length 2 and the Loewy length is automatically 3 . Now consider the case of 3 vertices. By Proposition 1.6 there is no loss in generality in assuming that $Q$ is strongly connected, for otherwise we can reduce the question of the finiteness of the global dimension to the case of 2 vertices and use 2.8 .

Proposition 2.9. Let $A=K Q / I$ be a 0-relations algebra of Loewy length 3 , where $Q$ is strongly connected with 3 vertices. Then $\operatorname{det} H=1$ implies that I can be generated by $\rho$, where $\rho$ consists of paths of length 2 .

Proof. It is assumed that there is a "bad" relation $a_{1} a_{2} a_{3}=0$ with $a_{1} a_{2}, a_{2} a_{3} \neq 0$. There are three configurations possible.

(i)

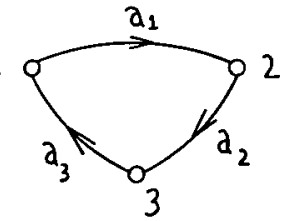

(iii)

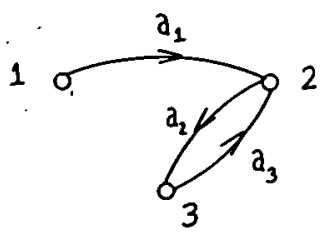

(ii)

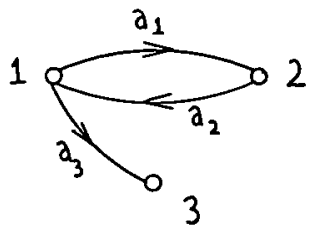

(A fourth case,

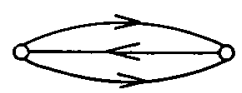

is already excluded by 2.8 .) 
In (i) the terms of $H$ just using $x_{1}, x_{2}$ and $x_{3}$ would be

$$
\left[\begin{array}{ccc}
1 & x_{2} x_{3} & x_{3} \\
x_{1} & 1 & u \\
x_{1} x_{2} & x_{2} & 1
\end{array}\right] \text {, where } u=x_{3} x_{1} \text { or } 0
$$

The determinant is $1+x_{1} x_{2}^{2} x_{3} u-x_{1} x_{2} x_{3}-x_{2} u \neq 1$.

(Note if $Q$ looks like (i) and det $H=1$ then exactly one of $a_{1} a_{2}, a_{2} a_{3}, a_{3} a_{1}$ is $\neq 0$.)

(ii) Since $Q$ is strongly connected, there is

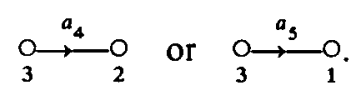

Consider first $a_{4}$. By (i) exactly one of $a_{2} a_{3}, a_{3} a_{4}, a_{4} a_{2}$ is $\neq 0$. Hence $a_{3} a_{4}=a_{4} a_{2}=0$; also by $2.7, a_{2} a_{1}=0$. The corresponding part of $\operatorname{det} H$ is

$$
\left[\begin{array}{ccc}
1+x_{1} x_{2} & x_{2} & 0 \\
x_{1} & 1 & x_{4} \\
x_{3} & x_{2} x_{3} & 1
\end{array}\right]=1-x_{1} x_{2}^{2} x_{3} x_{4} \neq 1
$$

With $x_{5}, a_{2} a_{1}=0$, by 2.7 , and similarly exactly one of $a_{3} a_{5}$ or $a_{5} a_{3}=0$. Put $u=x_{3} x_{4}$ or $0, v=x_{5} x_{3}$ or 0 . Let $w=x_{5} x_{1}$ or 0 .

$$
\left[\begin{array}{ccc}
1+x_{1} x_{2}+u & x_{2} & x_{5} \\
x_{1} & 1 & w \\
x_{3} & x_{2} x_{3} & 1+v
\end{array}\right]= \begin{cases}1+x_{1} x_{2} x_{3} x_{5}-x_{2} x_{3}^{2} x_{5} w-x_{1} x_{2}^{2} x_{3} w & \text { if } u \neq 0 \\
1+x_{1} x_{2} x_{3} x_{5}-x_{1} x_{2}^{2} x_{3} w & \text { if } v \neq 0\end{cases}
$$

$\neq 1$, regardless of $w$.

(iii) There is

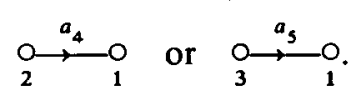

With $x_{4}$ there are two choices, either $x_{1} x_{4}=0$ or $x_{4} x_{1}=0$. Put $u=x_{1} x_{4}$ or $0, v=x_{4} x_{1}$ or $0, w=x_{3} x_{4}$ or 0 .

$$
\begin{aligned}
{\left[\begin{array}{ccc}
1+u & x_{4} & w \\
x_{1} & 1+x_{2} x_{3}+v & x_{3} \\
x_{1} x_{2} & x_{2} & 1
\end{array}\right] } & =\left\{\begin{array}{cc}
1+x_{1} x_{2} x_{3} x_{4}-x_{1} x_{2}^{2} x_{3} w & \text { if } u \neq 0 \\
1+x_{1} x_{2} x_{3} x_{4}-x_{1}^{2} x_{2} x_{4} w & \\
-x_{1} x_{2}^{2} x_{3} w & \text { if } v \neq 0
\end{array}\right. \\
& \neq 1 .
\end{aligned}
$$


With $x_{5}, x_{2} x_{5}=x_{5} x_{1}=x_{3} x_{2}=0$ by (i). We get

$$
\left[\begin{array}{ccc}
1+x_{1} x_{2} & 0 & x_{5} \\
x_{1} & 1+x_{2} x_{3} & x_{3} \\
x_{1} x_{2} & x_{2} & 1
\end{array}\right]=1+x_{1} x_{2}^{2} x_{3} \neq 1 \mathrm{n}
$$

Corollary 2.10. Let $A=K Q / I$ be a 0 -relations algebra where $Q$ has 3 vertices. Assume further that the Loewy length of $A$ is 3. Then if $H$ is the G-graded Cartan matrix, gl $\operatorname{dim} A<\infty$ iff $\operatorname{det} \boldsymbol{H}=1$.

Proof. This follows from 1.5, 2.8, 2.9, and 2.6.

The next example shows that for 4 vertices, Theorem 2.6 does not cover all cases of Loewy length 3 .

\section{Example 2.11.}

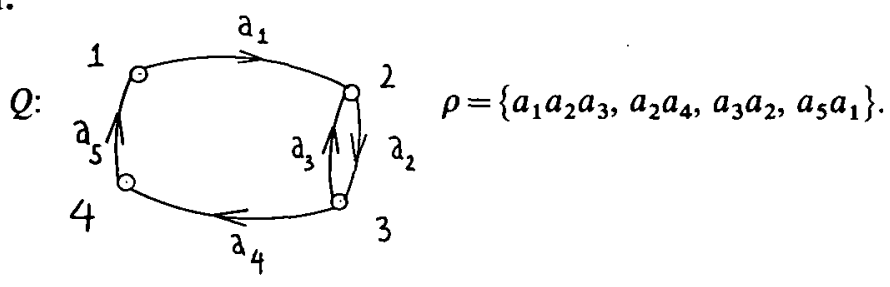

The corresponding algebra is of Loewy length 3 and global dimension 5 . Of course $\operatorname{det} H=1$.

To conclude, the following example is one where the algebra has a $\mathbb{Z}$-grading whose Cartan matrix has determinant 1 , but which is of infinite global dimension. However the $G$-grading Cartan matrix detects this.

Example 2.12.

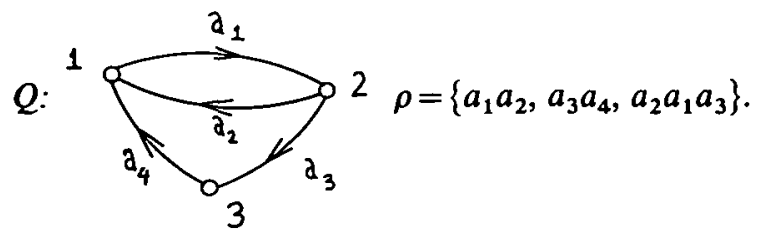

The most natural $\mathbb{Z}$-grading has Cartan matrix

$$
\left[\begin{array}{ccc}
1 & x & x \\
x & 1+x^{2} & x^{2} \\
x_{2} & x & 1+x^{3}
\end{array}\right], \quad \text { with determinant } 1
$$

There are other $\mathbb{Z}$-gradings also yielding matrices with determinant 1 , but $\operatorname{det} H \neq 1$, as 
follows by computation or by 2.9. (This example was found by Fuller and Zimmermann-Huisgen and is presented here as a 0-relations algebra.)

Note added in proof. T. Belzner, in a thesis being written at the University of Passau, has used the gradings due to Green and has obtained Theorem 2.6 without the restriction on the Loewy length.

\section{REFERENCES}

1. W. D. Burgess, K. R. Fuller, E. Voss and B. Zimmermann-Huisgen, The Cartan matrix as an indicator of finite global dimension for artinian rings, Proc. Amer. Math. Soc. 95 (1985), 157-165.

2. P. M. Cohn, Free Rings and their Relations (Academic Press, London and New York, 1971).

3. S. Eilenderg, Algebras of cohomologically finite dimension, Comment. Math. Helv. 28 (1954), $310-319$.

4. R. Fossum, P. Griffith and I. Reiten, Trivial extensions of abelian categories and applications to rings: an expository account, in Ring Theory (Ed. R. Gordon) (Academic Press, New York, London, 1972).

5. P. Gabriel, Unzerlegbare Darstellungen I, Manuscripta Math. 6 (1972), 71-103.

6. E. L. Green, Graphs with relations, coverings and group-graded algebras, Trans. Amer. Math. Soc. 279 (1983), 297-310.

7. E. L. Green, D. Happel and D. Zacharia, Projective resolutions over artin algebras with zero relations, Illinois J. Math. 29 (1985), 180-190.

8. G. V. Wilson, The Cartan map on categories of graded modules, J. Algebra 85 (1983), 390-398.

9. D. Zacharia, On the Cartan matrix of an artin algebra of global dimension two, J. Algebra 82 (1983), 353-357.

Department of Mathematics

UNIVERSITY OF OTTAWA

Ottawa, Canada

K1N 6N5 